\title{
Erratum: Leading higher-derivative corrections to Kerr geometry
}

\section{Pablo A. Cano and Alejandro Ruipérez}

Instituto de Física Teórica UAM/CSIC,

C/Nicolás Cabrera, 13-15, C.U. Cantoblanco, 28049 Madrid, Spain

E-mail: pablo.cano@uam.es, alejandro.ruiperez@uam.es

ERRATUM TO: JHEP05(2019)189

ARXIV EPRINT: 1901.01315

There was an error when copying eqs. (4.41), (4.42) and (4.43) from the Mathematica file. The error does not propagate and, in particular, the figures are not affected. The correct expressions for eqs. (4.41), (4.42) and (4.43) are the following

$$
\begin{aligned}
\Delta \omega_{ \pm}^{(1)}= & \frac{4397}{65610 \sqrt{3}} \pm \frac{20596 \chi}{295245}+\frac{1028803 \chi^{2}}{14467005 \sqrt{3}} \pm \frac{45262543 \chi^{3}}{3906091350}-\frac{3685587061 \chi^{4}}{328111673400 \sqrt{3}} \\
& \mp \frac{110632797883 \chi^{5}}{5413842611100}-\frac{910228742414947 \chi^{6}}{17151053391964800 \sqrt{3}} \mp \frac{15449837941866829 \chi^{7}}{401334649371976320}+\mathcal{O}\left(\chi^{8}\right), \\
\Delta \omega_{ \pm}^{(2)}= & \mp \frac{131 \chi}{5103}-\frac{11047 \chi^{2}}{381024 \sqrt{3}} \mp \frac{9491513 \chi^{3}}{1388832480}-\frac{19022279 \chi^{4}}{925888320 \sqrt{3}} \\
& \mp \frac{353193404087 \chi^{5}}{23099061807360}-\frac{2452581602509 \chi^{6}}{63522419970240 \sqrt{3}} \mp \frac{5958423964756267 \chi^{7}}{222963694095542400}+\mathcal{O}\left(\chi^{8}\right), \\
\Delta \omega_{ \pm}^{(\mathrm{ev})}= & \frac{20}{2187 \sqrt{3}} \pm \frac{320 \chi}{19683}+\frac{26749 \chi^{2}}{1928934 \sqrt{3}} \mp \frac{12967 \chi^{3}}{104162436}-\frac{4415651 \chi^{4}}{1249949232 \sqrt{3}} \\
& \mp \frac{3101153 \chi^{5}}{937461924}-\frac{33998483 \chi^{6}}{6629195034 \sqrt{3}} \mp \frac{18127693795 \chi^{7}}{6682228594272}+\mathcal{O}\left(\chi^{8}\right),
\end{aligned}
$$

Open Access. This article is distributed under the terms of the Creative Commons Attribution License (CC-BY 4.0), which permits any use, distribution and reproduction in any medium, provided the original author(s) and source are credited.

\section{References}

[1] P.A. Cano and A. Ruipérez, Leading higher-derivative corrections to Kerr geometry, JHEP 05 (2019) 189 [arXiv:1901.01315]. 